\title{
USEFULNESS AND POTENTIAL BENEFITS OF ANALYZING NEW MEDIA FROM THE PERSPECTIVE OF L. MANOVICH'S SOFT TECHNOLOGICAL DETERMINISM - THE CASE OF INSTAGRAM AND SMARTPHONE.
} Marcin Wielgosz ${ }^{1}$

\section{Abstract}

The article is an attempt to confront Lev Manovich's soft technological determinism with two contemporary media - the smartphone and the mobile application called Instagram. The analysis is based on the characteristics of a term called "new media" identified by Manovich, with emphasis on variation and cultural transcoding.

The verification of accuracy and the use of the soft technological determinism in the context of selected new media has varied by the discourse on contemporary new media and constitutes an interesting point of view. The evolution and the development of both variation and cultural transcoding (two elements conditioning their universality which continually shows an upward trend) regarding mobile media, give an opportunity to forecast their productive potential. ism

Keywords: new media, smartphone, new media theories, cultural transcoding, technological determin-

\section{Intorduction}

The object of the article is to verify Lew Manovich's theory of the new media included in the book The Language of New Media. It is believed that Manovich's soft technological determinism potentially allows to deepen the understanding of the specifics of new media and also makes it possible to predict their future development. To see if Manovich's theory has not lost its relevance, the authors decided to confront the features of new media identified by Manovich with the examples of popular media created after 
the first publication of The Language of New Media - mobile device: Smartphone and mobile application: Instagram.

From a sociological perspective, both Smartphone and Instagram are the subjects of many studies because both of those media have a substantial impact on society and its communication habits. The look at the media through the prism of soft technological determinism will allow us to confirm if the newly emerging media still are the subjects of its logic. If it is confirmed, then, it means that we can try to predict further directions of the development of new media following their inherent traits identified by Manovich.

\section{Methodology}

Manovich identified five features deriving from the technological conditions of the new media. Among them: numerical representation, modularity, automation, variation, cultural transcoding. The first three, since we are talking about a device operating under the control of a digital operating system and a mobile application, are the basis for the functioning of these media. Despite the fact that variation and cultural transcoding result from the first three characteristics they are much more abstract because they refer to the transfer of technology logic to the medium and its content layer. Therefore, the relevance of Manovich soft technological determinism depends on the continuing relevance of that two most important features of the new media identified by him. We were searching the continuous relevance of Variation in the changes of software caused by the creation of a smartphone. We found its example in the evolution of the web interface. The impact of technology on the content of the medium, i.e. cultural transcoding was looked through the analysis of the mobile application - Instagram.

\section{The research landscape of new media.}

Written materials related to the theory of new media put particular emphasis on the complexity of this term, and it often results in adopting completely different points of view by its researchers. It also leads to treating various aspects of new media as superior and causes not only conflicting categorization but also and a diverse (though not always antagonistic) approach to the issue. Social processes initiated by the creation of media such as the computer or the Internet are crucial to many authors, and sometimes they are so groundbreaking that they are treated as new media or are the basis for opening a new era in the media history. M. Lister, J. Dovey, S. Giddings, I. Grant, K. Kelly [2009] in the New Media study claim that the popularity of the term new media originates from the widespread feeling of the sublimity of rapid changes that occurred in the field of communication and media in the 1980s. We can assume that these authors look the new media from the sociological perspective, and the social dimension of these media is perceived as crucial. A similar approach is presented by M. Castells [2008], who in his work called Network Society comprehensively captures a broad spectrum of social changes related to the information technology revolution. The rise and development of social media also contributed to the domination of sociological topics in the current media discourse. According to Paul Levinson [2010] the most popular of them, i.e. blogging, YouTube, Wikipedia, Digg, MySpace, Facebook, Twitter and Second Life, have changed the world to the same degree as computers or the Internet. The quality upgrade that he saw after these media were created prompted him to separate them and create a category of the New New Media.

The analysis of the new media, focused mainly on their social dimension, allows us to better understand the media reality, and consequently its social aspect. Although they are currently described in the written materials, let us remember that they are not the only available sources of knowledge. Their supplement is a technological paradigm. The confrontation of its theoretical assumptions with the widely used new medium called a smartphone will allow us to come to the conclusions and help us to further understand the usefulness of the postulates of technological determinism in the understanding of 
the dynamically changing world of new media.

\section{Manovich's soft technological determinism and its features of new media.}

L. Manovich in his best-known study The Language of New Media presents his perspective of media history interpretation called "technological determinism." Lev's reasoning is based on the assumption that technology has the fundamental and decisive influence on shaping the society's customs. Manovich describes the role of the new media logic and its specific language. Understanding and adopting this specific logic allow us to use the full potential of new media, and its versatility helps the society in adapting its language. This is not a relatively new concept as such deterministic point of view was already present in the science of media long before the dissemination of the so-called new media. Harold Innis [1951], and his continuer was Marshall McLuhan [1964] were Manovich's progenitors. Both of them shared the same view that the medium and communication technology dominating at a given time in a given area shapes social and political processes (also because the medium is appropriated by the centers of power and used to distribute knowledge). Regarding Manovich's theory, the concept of determinism is combined with the adjective "soft" because it stresses the importance of sociological factors related to the functioning and shaping of those media, although it does not clearly state when a given medium can be called 'new.'

Manovich, contemplating the definition of the concept of new media, based his common understanding on the popular press. He stresses that narrowing the understanding of new media to the means of content distribution (to which it often brings the notion of common knowledge) does not exhaust its content and makes the impact of new media on the entire culture impossible to understand. The author draws attention to the possibility of using new media not only to create but also to save and process objects. The starting point in Manovich's [2006, p. 82] reasoning is the assumption that new media are the result of the development of computing and media technologies. The synthesis of the intersection of those two fields describes the ability to translate all available media into the language of discrete data, read by computers. As a result, it allows processed media to be named as new.

Describing the features of new media is primarily focused on their technical dimension and the impact on the identity of those media. It specifies the following rules for their objects:

- Numerical representation - regardless of whether the new media object was created on the computer or if it was created in the process of converting from an analog form, it must be possible to describe it in a mathematical language that can always be subjected to the process of tooling.

- Modularity - resulting from the "fractal structure of new media," meaning that elements of new media, regardless of whether we are talking about images, sounds, shapes, etc., can always be treated as preserving their own collection of discontinuous samples that build a larger whole but can still be processed individually.

- Automation - this feature stems from the two mentioned above and indicates the ability to automate many processes related to the creation, processing, and sharing of new media.

- Variation - as an alternative term for this definition Manovich also mentioned fluidity and variability and described it as the occurrence of objects of new media in different versions, which are not necessarily inverted or composed by man but also by a computer - so variation is possible due to modularity and automation.

- Cultural transcoding - recognized by Manovich as the most important consequence of computerization of the media, it indicates the mutual influence of the computer layer (that is, computer logic and thus a specific ontology, epistemology, and pragmatics) on the cultural layer of the medium [Manovich, 2006, pp. 91-118]. 
It is hard to deny the logic behind this reasoning, even after adopting an intellectual attitude other than technological determinism. The features mentioned by Manovich indeed characterize the objects of new media, but the question of whether the medium should be categorized as new is a problem that remains unsolved. It is difficult to find a numerical representation in media before the IT revolution or to automate media in the dimension in which we encounter those phenomena in broadly understood information technology. Thinking about the media in terms of the language proposed by the author of The Language of New Media directs us to search for new devices and technical possibilities resulting from their creation.

Since the first release of The Language of New Media, one of the most groundbreaking medium created, mainly due to its universality, was the smartphone. It is described as an advanced multimedia device that combines functionalities of a laptop, a phone, and a camera. Currently used smartphones are generally characterized by high computing power while having a compact size. Let's try to confront this medium with the features of the new media, postulated by Manovich. Numerical representation, modularity, and automation are the inherent features of every operating system that supports such device. It seems, however, that the creation of a smartphone and the smartphoneization process itself have contributed to the exceptional intensification of both variation and cultural transcoding. In the light of data related to the popularity of these devices, the development of both features is not surprising and seems almost unavoidable. Understanding the basic statistics on the prevalence of the use of smartphones will illustrate the scale in which a specific language of the new medium influences the society. Analysis of variation and cultural transcoding in the context of this medium also allows looking more closely at its impacting abilities.

\section{The Variation and the smartphones.}

It is estimated that currently, more than 2.41 billion people worldwide are smartphone users, and by the end of 2018, it is estimated that more than one-third of the world's population will be using smartphones [Statista, Number of smartphones]. The numbers associated with the increase in browsing the Internet are just as impressive. In 2016 , approximately $43 \%$ of website global traffic was generated on mobile devices, and it has increased by 35\% compared to 2015 [Statista, Percentage]. In between years 2016 and 2017, the number of unique mobile users grew by at least 222 million which led to a $6 \%$ increase [Statista, Number of smartphones]. Also, it is estimated that a smartphone user uses his or her device 4 hours every day [Hackernoon, 2017]. The quoted statistics allow us to consider smartphones as all-time universal devices. Can we imagine their popularity without developing the variation of new media objects? If we take into account the context of using a traditional computer or a laptop versus the usage of a smartphone, it becomes clear that the answer is 'No'. A perfect example of variation is the creation, development and continuous optimization of the interfaces operated on the touchscreen. We have already pointed out that a smartphone is usually compact in size - this is mainly because one of its essential functions is the ability to make calls and send text messages, so the user should be able to have the device with him or her the whole time. The size of the screen affects how it is operated, so it also impacts the interface. This is due to very pragmatic reasons - the construction of the desktop website is very extensive, and the mouse cursor in the context of the monitor screen is a tool much more precise than the human finger controlling the small screen of the smartphone. The computing abilities of today's smartphones and the accessibility to the Internet allow us to perform the same actions available on our regular-sized computers. Optimal and comfortable use of smartphones is possible thanks to the interface adapted to its diagonal screen. In reality, it means that websites nowadays have been optimized to fit the smartphone's screen - this relates to the graphics, menus, and site's content. Research shows that page loading time influences our decision whether to stay or leave it so the faster 
the page displays correctly on the mobile device, the greater the chance that the user will stay and perform the desired action [Majchrzyk, 2017]. It is a common knowledge that users surfing the Internet on their phones most often look for store opening hours, company's information such as phone numbers and addresses, etc., so user's satisfaction depends on the exposure and availability of that information, which later results in the increase of website's popularity. The use of the variation of new media in the context of smartphones has made it possible to adapt this medium to social expectations better. Until recently, the user could choose whether he wanted to use the mobile or desktop version of a website, but nowadays they tend to adapt themselves to the screen on which they are displayed. This functionality is called Responsive Web Design, and it means that there is an algorithm responsible for communication between the device and the server which enables the screen size identification on which the page will be displayed, and then it adjusts its graphic design and interface. Website optimization from the user's point of view happens automatically, but it is predetermined and designed for particular screen diagonal ranges, and may include a choice between a classic (desktop) interface and a mobile one depending on the diagonal of the screen on which it is displayed. Designing responsive websites is becoming a standard process, which is understandable as it has a positive impact on the overall using experience. Here is a perfect example of the variation of new media - Responsive Web Design technology is supported by Google so it is understandable that it has a substantial impact on indexing pages in Google search engine, which is now the technological standard. Its popularity is growing and considering the tendencies related to mobile internet consumption and constant smartphoneization it is no wonder that this website is known worldwide.

\section{Instagrams reality created by the take-anywhere mobile camera.}

According to Manovich cultural transcoding is being regarded as the most important consequence of computerization of the media. The development of variation related to the popularization of smartphones and the need to adapt network resources to their capabilities proves the existence of a specific logical program that is used on mobile devices. The newly created logic for the smartphone contributed to the creation and development of the most well-known mobile application for photo hosting which is breaking popularity records across the world and is called Instagram. This application was created in 2010 and was free of charge on IOS mobile operating system (initially, it could only be used on Apple brand smartphones). The application allows registered users to share photos and videos and it also gives the possibility to apply filters, adjust the general settings and mark not only the place where the picture was taken but also profiles of other registered users. Statistics show that taking photos and recording short films are the most often carried out activities by smartphone users - it is only surpassed by browsing the Internet [Marketing Charts, 2016]. The very nature of the photos is the subject to the logic of the medium - that is the smartphone and the context in which it is used. Considering the above, it is, in fact, a small camera that is always with us, used for documenting our lives. The photographs that are made are not always of good quality, and they often don't present high artistic values, but Instagram was designed to accommodate this logic. At the time of its creation, the service enabled sharing photos in the resolution not higher than $640 \times 640$, which determined their low quality but surprisingly it did not prevent the service from becoming more and more popular. ${ }^{2}$ Currently, it is ranked 3rd regarding the number of users of social networking sites in the world with the number of active users, exceeding the amount of 800 million [Kallas, 2017]. With this in mind, we can ask ourselves where its popularity came from? It seems that the answer can be found both in the technical features of the application and in its social dimension. 
Coming back to L. Manovich's logic, we will focus on technical aspects in which we can find the impact of the application on the shared content, i.e. cultural transcoding.

Let us identify the areas in which the most important new media feature is reflected in the Instagram mobile application.

- The mobile nature of the application and its functions.

Instagram has been programmed to be easy to use when people are on the move. Taking a photo requires from the user only one operation. Publishing it, even if preceded by an additional change of the settings, takes just a few seconds. Currently, it is also possible to participate in real-time broadcasts, where observers can join a chat, or they can comment on the viewed images.

- Users Friendly Interface.

The application's interface is simple and intuitive, which makes it easy to use. This may not seem visible at first glance, but the fact that users do not think about the interface while using the application means that it is well designed and it also proves its most desirable feature - intuitiveness [Krug, 2017, pp. 10-20]. The graphic layout of the application along with the interface, on the whole, are coherent, aesthetic and easy to use. Because of this even less interesting photos gain on attractiveness.

- Characteristic functionalities.

The technical possibilities that creators enabled in the application and which are vastly popular have a significant impact on its success. Probably the most important one is the possibility of pre-processing a photo using graphics filters. They alter the colors, temperature, exposure and even its design (the photo can be aged by applying the correct filter). The popularity of filters is driven by the desire to present each picture most attractively at the expense of authenticity, which becomes debatable. Filters are widely used by Instagram users as a method of editing and improving the reality presented in the pictures and are another manifestation of cultural transcoding. ${ }^{3}$

\section{- Categorization of content}

The only option currently available for categorizing the content on Instagram is using hashtags. The word is made of two English words - 'hash' which stands for the sign '\#' and 'tag' for a marker. A hashtag is any word preceded by a '\#' sign used to group and categorize content on Instagram. It was initially used to classify content on the popular IRC platform in the 1980s. The creators of Instagram adapted this categorization method, and the community started coming up with their own, original hashtags. The use of a hashtag does not always have to be limited to the categorization of content (although it is still its primary role), it can also perform other functions such as expressing the desire to manifest belonging to a given group, identifying with specific values, showing aspirations, etc. The hashtags from Instagram have penetrated the colloquial language and youth pop culture (e.g., they are commonly seen them in hip-hop texts).

Instagram has been primarily programmed for one medium - a smartphone. From the perspective of L. Manovich described in The Language of New Media, the smartphone functions in modern society as a new medium thanks to the smartphone-adapted applications. What makes it unique is not the fact that it was made several years after the first edition of the book, in which Lev conceptualized the paradigm of soft technological determinism, but that it meets the assumptions of this theory. The relationship between the medium and society in the case of cultural variation and transcoding often occurring in the form of feedback (which is exemplified by the responsiveness of websites) complements the sociological theories and still allows a better understanding of the functioning of media reality.

More about the popularity of filters on Instagram, See. https://www.canva.com/learn/popular-instagram-filters/ [online: December 3, 2017]; 


\section{Discussion}

Concerning the smartphone as well as the mobile application Instagram, features of new media identified by Manovich have been proved which also confirms the further usefulness of the concept of soft technological determinism in explaining the reality of new media and its potential future development. As it was previously mentioned the contrary point of view is social determinism. Authors of this conception emphasize that society makes all of the technological changes which come from the economic system. It is more the society that influences the shape of technology than the reverse. Among the most well-known representatives of this trend, M. Castells [2008], P. Levy [1998], M. Lister and others [2008], and P. Levinson [2010] can be mentioned. It should be also emphasized that individual authors do not deny the impact of technology on society and its communication habits, but simply do not admit its primary role. If we take in to account, that we considered the further use of technological determinism as a still-current approach that broadens our view of technology, but not the only proper, theories of social determinism do not contradict the basic thesis of work.

\section{Conclusion}

New media that are continually emerging and evolving can still be analyzed from the point of view of the characteristics of the new media identified by Manovich. Numerical representation, modularity, and automation are inseparably related to all digital media controlled by operating systems. Further existence and evolution of variation and cultural transcoding were not apparent. The responsive web interface is a practical realization of the variation resulting from the need to adjust the display of web pages to different screen sizes. Indeed, further research on this topic is recommended, because it is certainly possible to find more examples of variations resulting from the growing use of smartphones in the Internet consumption.

Instagram turned out to be a great example of cultural transcoding. The impact of a specific logic of photography taken by the mobile phone camera as a way to express oneself but also taking pictures at all and their functions in social reality seem to be very wide. Confirmation of technological factors affecting the medium content layer (the example of the Instagram) at the same time confirms the claim that "the medium is the message".

We consider that conducting further research in the field of soft technological determinism is reasonable and potentially very beneficial for a better understanding of the media and the possibilities of future development.

\section{References:}

CASTELLS M. (2008), Społeczeństwo sieci, Warszawa, Wydawnictwo Naukowe PWN.

INNIS H, (1951), The Bias of Communication, Toronto, University of Toronto Press.

KALLAS P. (2017), Top 15 most popular social networking sites, [online: December 3, 2017], https://www. dreamgrow.com/top-15-most-popular-social-networking-sites/, compare: https://www.statista.com/statistics/253577/number-of-monthly-active-instagram-users/ [online: December 13, 2017].

KRUG S. (2017), Don't make me think: A Common Sense Approach to Web Usability, California, New Readers Publishing.

LISTER M., DOVEY J., GIDDINGS S., GRANT I., KELLY K. (2009), Nowe media, Kraków, Wydawnictwo Uniwersytetu Jagiellońskiego.

MAJCHRZYK Ł. (2017), Cechy dobrze zaprojektowanej strony mobilnej, [online: December 3, 2017], https:// mobirank.pl/2014/07/17/cechy-dobrze-zaprojektowanej-strony-mobilnej/.

MANOVICH L. (2006), Język nowych mediów, Warszawa, Wydawnictwa Akademickie i Profesjonalne. 
MARKETING CHARTS (2016), These Are Our Most Popular Smartphone Activities, [online: December 3, 2017], https://www.marketingcharts.com/industries/media-and-entertainment-71063.

MCLUHAN M. (1964), Understanding Media: The Extensions of Man, New York McGraw-Hill.

Statista, Number of smartphone users worldwide from 2014 to 2020 (in billions), [online: December 3, 2017], https://www.statista.com/statistics/330695/number-of-smartphone-users-worldwide/.

Statista, Percentage of all global web pages served to mobile phones from 2009 to 2017, [online: December 3, 2017], https://www.statista.com/statistics/241462/global-mobile-phone-website-traffic-share/.

HACKERNNON (2017), How Much Time Do People Spend on Their Mobile Phones in 2017?, [online: December 3, 2017], https://hackernoon.com/how-much-time-do-people-spend-on-their-mobile-phones-in-2017-e5f90a0b10a6.

LEVY P. (1998), Becoming Virtual, New York: Plenum Trade 1998,

LEVINSON P. (2010), Nowe Nowe Media, Kraków 2010 